\title{
Boundary Effects in Stokes' Problem with Melting
}

\author{
Arup Mukherjee and John G. Stevens \\ Montclair State University, Montclair NJ 07043, USA \\ \{mukherjeea, stevensj\}@mail.montclair.edu
}

\begin{abstract}
We discuss the use of the computer algebra system Maple (B) to analyze the heat transport when a heated sphere melts its way through a solid medium. The combined power of symbolic computation and numerical simulation available in Maple is used to analyze a model in which the molten layer around the moving sphere is augmented by a surrounding thermal-layer. In particular, we carefully study the effects of imposing a variety of boundary conditions.
\end{abstract}

\section{Introduction}

The power of large-scale simulations has had a major impact in advancing the understanding of physical processes in a multitude of applications. In many circumstances, the careful analysis of the model and an understanding of the effects of particular model parameters can create an efficient feedback mechanism which iteratively improves the model and provides insights into the underlying physical phenomena. Using different approximations for unknowns in a model, or comparing the effects of different boundary conditions are useful tools in this feedback cycle. In this paper, we use the power of Maple to advance the understanding of a model for Stokes' problem with melting. In particular, we study the effects of changing boundary conditions that affect the basic unknowns of the model. The classical Stokes' problem calculates the drag on a sphere moving at a constant velocity through a viscous, fluid medium. "With melting" assumes that the sphere maintains it surface temperature above that of the surrounding medium due to some internal heat source, the heat from the sphere is sufficient to melt a region around it, and the sphere falls through the melted region until it attains a steady state velocity. The model describes situations ranging from magma migration and core formation to that of a run-away nuclear reactor melting its way through the earth [3]. In section 2 we introduce the integral balance method and its innovative use in Stokes' flow with melting proposed by Emerman and Turcotte 1]. We study the effects of imposing improved boundary conditions for the velocity, and demonstrate that this leads to an improved estimate for the melt-layer. Section 3 highlights the differences between the melt-layer model and a more accurate two-layer approach [2] where a phenomenological thermal layer is added beyond the melt layer. The boundary condition for the temperature profile used in the melt-layer model of section 2 leads to certain anomalies which 
are resolved by the two-layer model. The thickness of the thermal-layer is obtained as a series approximation by re-applying the integral balance method on the thermal-layer. This approximation for the thermal-layer thickness is needed to impose the correct boundary condition for the temperature profile in the meltlayer. A repeat of the integral balance method in the melt-layer then yields the melt-layer thickness.

\section{Integral Balance Method: Melt-Layer Model}

In the integral balance method, it is assumed that heat is transported over some distance $\delta$, and the heat conduction equation is integrated over this thermal layer thickness to obtain the integral heat balance. Appropriate boundary conditions and a trial function of a spatial variable are assumed for the temperature profile, leading to an expression involving the spatial variable and $\delta$. Substituting this expression into the integral heat balance leads to a differential equation for $\delta$ which is solved to obtain an expression for the thermal layer thickness. Finally, the temperature profile is obtained through direct substitution. Emerman and Turcotte [1] employed the integral balance method to obtain an expression for the non-dimensional melt layer thickness, $\delta(\theta)$. The relevant geometry is displayed in Figure 1. Using the non-dimensional variables $\theta=x / R, \mathbf{y}=y / R, \delta=d / R, \mathbf{u}=u / u_{0}, \mathbf{v}=v / u_{0}$, and $\mathbf{T}=\left(T-T_{m}\right) /\left(T_{0}-T_{m}\right)$, the Navier Stokes equation in lubrication form with boundary conditions $\mathbf{u}(0)=$ $\mathbf{u}(\delta)=0$ leads to $\mathbf{u}(\mathbf{y})=-(3 \mathbf{y} \sin \theta)(\mathbf{y}-\delta) / \delta^{3}$. The Stefan number is defined as Ste $=\frac{c_{p}\left(T_{0}-T_{m}\right)}{L+c_{p}\left(T_{m}-T_{\infty}\right)}$ where $c_{p}$ is the specific heat capacity of melt, $L$ is the latent heat of fusion, and $T_{\infty}$ is the ambient temperature of the medium and the Peclet number is defined as $\mathrm{Pe}=u_{0} R / \kappa$, where $\kappa$ is the thermal diffusivity of melt. Ste and $\mathrm{Pe}$ form the dimensionless groups for the problem in non-dimensional form. Assuming a quadratic profile for temperature $\mathbf{T}(\mathbf{y})$ and imposing the conditions $\mathbf{T}(0)=1, \mathbf{T}(\delta)=0$ and $\partial \mathbf{T} / \partial \mathbf{y}=-$ Pe $\cos \theta /$ Ste, when $\mathbf{y}=\delta$ yields the temperature profile $\mathbf{T}(\mathbf{y})=1+\mathbf{y}\left(\frac{\mathrm{Pe}}{\mathrm{Ste}} \cos \theta-\frac{2}{\delta}\right)+\mathbf{y}^{2}\left(\frac{1}{\delta^{2}}-\frac{\mathrm{Pe}}{\mathrm{Ste}} \frac{\cos \theta}{\delta}\right)$. Substituting the expressions for $\mathbf{u}(\mathbf{y})$ and $\mathbf{T}(\mathbf{y})$ into the integral heat balance and solving the resulting differential equation for $\delta(\theta)$ produces the melt-layer thickness as $\delta_{\mathbf{E T}}(\theta)=\delta_{\mathbf{E T}}(0) \sec \theta=f($ Ste $) \sec \theta /$ Pe, where $f(z)=0.25(-3 z-20+$ $\left.\sqrt{9 z^{2}+280 z+400}\right)$. Complete details of the derivation in this form can be found in [1, 2]. The energy equation for this model leads to $\partial^{2} \mathbf{T} / \partial \mathbf{y}^{2}=0$ when $\mathbf{y}=0$, which yields the approximation $\delta=\delta_{\mathbf{E T}}(\theta)=(\mathrm{Ste} / \mathrm{Pe}) \sec \theta$. It is easily verified that as Ste $\rightarrow 0, f($ Ste $) \rightarrow$ Ste. Thus for small values of Ste, $\delta_{\mathbf{E T o}} \approx \delta_{\mathbf{E T}}$ while always satisfying $\delta_{\mathbf{E T o}}>\delta_{\mathbf{E T}}$.

\subsection{Boundary Conditions for Velocity}

The boundary condition $\mathbf{u}(\delta)=0$ is a good approximation under the assumption $\delta<<1$, but is clear that the correct boundary condition at the boundary of the melt-layer is $\mathbf{u}(\delta)=-\sin \theta$. Solving for the velocity using this improved boundary condition results in $\mathbf{u}(\mathbf{y})=-3 \mathbf{y} \sin \theta\left(\mathbf{y}-\delta+\mathbf{y} \delta-\frac{2}{3} \delta^{2}\right) / \delta^{3}$. Using this expression for $\mathbf{u}(\mathbf{y})$ and assuming the temperature profile given above, a modified 




Fig. 1. Geometry for melt-layer and two-layer Stokes' Problem with Melting. The variables are discussed in detail in section 2

Table 1. Relative Percentage error $\left|\frac{\delta_{\mathbf{M}}(\theta)-\delta_{\mathbf{E T}}(\theta)}{\delta_{\mathbf{E T}}(\theta)}\right| \times 100$ for fixed Ste $=0.5$ (left) and fixed $\mathrm{Pe}=10$ (right)

\begin{tabular}{lccccc||cccccc} 
colatitude & \multicolumn{1}{c}{$1 \leq \mathrm{Pe} \leq 50$} & with & Ste $=0.5$ & \multicolumn{3}{c}{$0.1 \leq$ Ste $\leq 2.0$} & with $\mathrm{Pe}=10$ \\
\cline { 2 - 11 }$\Downarrow$ & 1 & 5 & 10 & 15 & 50 & 0.1 & 0.25 & 0.5 & 1.0 & 2.0 \\
\hline$\theta=\pi / 4$ & 2.93 & 0.63 & 0.32 & 0.21 & 0.06 & 0.02 & 0.09 & 0.32 & 0.96 & 2.52 \\
$\theta=\pi / 3$ & 5.74 & 1.32 & 0.68 & 0.45 & 0.14 & 0.04 & 0.21 & 0.68 & 1.95 & 4.77 \\
$\theta=\pi / 2.5$ & 0.13 & 0.65 & 1.94 & 4.98 & 10.67 & 13.26 & 3.67 & 1.94 & 1.32 & 0.41 \\
$\theta=\pi / 2.4$ & 17.24 & 5.19 & 2.79 & 1.92 & 0.60 & 0.20 & 0.98 & 2.79 & 6.81 & 13.84 \\
$\theta=\pi / 2.3$ & 23.58 & 8.00 & 4.45 & 3.09 & 0.98 & 0.37 & 1.67 & 4.45 & 10.06 & 19.02 \\
$\theta=\pi / 2.2$ & 34.40 & 13.97 & 8.23 & 5.85 & 1.94 & 0.88 & 3.45 & 8.23 & 16.61 & 28.27 \\
$\theta=\pi / 2.1$ & 54.71 & 29.72 & 19.64 & 14.75 & 5.43 & 3.28 & 10.04 & 19.64 & 32.82 & 47.32 \\
\hline
\end{tabular}

melt layer thickness, $\delta_{\mathbf{M}}(\theta)$, is easily computed. Unlike the melt-layer expressions $\delta_{\mathbf{E T 0}}(\theta)$ and $\delta_{\mathbf{E T}}(\theta)$ which become infinite as $\theta \rightarrow \pi / 2, \delta_{\mathbf{M}}(\pi / 2)$ is finite. Table 1 shows the relative percentage errors as a function of the Peclet number Pe and the Stefan number Ste as the colatitude $\theta$ changes. When Ste is fixed, For each value of $\mathrm{Pe}$, the errors increase as the colatitude $\theta$ approaches $\pi / 2$. Also, for any fixed value of $\theta$, the errors are a decreasing function of Pe. Again, for any fixed value of Ste, the errors increase monotonically as $\theta \rightarrow \pi / 2$, and for any fixed value of $\theta$, the errors increase with increasing Ste. The third column for both tables correspond to the non-dimensional group pair Ste $=0.5$ and $\mathrm{Pe}=10$. These values for Ste and Pe are very close to the specific example worked out in detail in [2]. In fact, if we assume that the sphere resembles a small rock penetrator of radius $3 \mathrm{~cm}$ melting its way through a typical rock like basalt, the relevant physical properties lead to Ste $=0.556$ and $\mathrm{Pe}=10.33$. For values of Ste and $\mathrm{Pe}$ in a range where our analysis makes sense, the inequality $\delta_{\mathbf{M}}<\delta_{\mathbf{E T}}<\delta_{\mathbf{E T 0}}$ always holds and the separation between $\delta_{\mathbf{M}}$ and $\delta_{\mathbf{E T}}$ increases as the equator is approached $(\theta=\pi / 2)$. 


\section{Integral Balance Method: Two-Layer Model}

The boundary condition $\partial \mathbf{T} / \partial \mathbf{y}=-(\mathrm{Pe} / \mathrm{Ste}) \cos \theta$ at $\mathbf{y}=\delta$ implies that the gradient of the temperature is zero at the equator. This condition is inconsistent with the temperature at this point being $T_{m}$ while the surrounding solid medium has a temperate $T_{\infty}$. An improved boundary condition at this melt/solid interface, based on the requirement that the latent heat required to melt the solid must be supplied by conduction, is $\partial \mathbf{T} / \partial \mathbf{y}=-(\mathrm{Pe} / \sigma) \cos \theta+\beta(\partial \mathcal{T} / \partial \mathbf{y})$, where $\beta=k_{s}\left(T_{m}-T_{\infty}\right) / k_{l}\left(T_{0}-T_{m}\right)$, and $\sigma=c_{p}\left(T_{0}-T_{m}\right) / L$ is similar to Ste but based on the actual latent heat, $\mathcal{T}=\left(T_{s}-T_{\infty}\right) /\left(T_{m}-T_{\infty}\right)$ is the non-dimensional temperature in the thermal-layer, and $k_{s}$ and $k_{l}$ represent the thermal conductivities for solid and liquid (melt) respectively. The geometry for the two-layer model is shown in Figure 1. If $\varepsilon(\theta)$ is the non-dimensional thermal layer thickness, the integral balance method described in section 2 can be applied to the thermal layer to obtain a series approximation for $\varepsilon(\theta)$, and $\mathcal{T}(\mathbf{y})$ is then computed by direct substitution. Finally, the resulting expression for $\mathcal{T}(\mathbf{y})$ is used in the boundary condition above and the integral balance method applied to the melt-layer to obtain its thickness, $\delta_{\mathbf{2} \mathbf{L}}(\theta)$. The salient differences between $\delta_{\mathbf{2} \mathbf{L}}$ and $\delta_{\mathbf{E T}}$ computed using $\mathbf{u}(\delta)=0$ are outlined in 2. The melt-layer thickness, $\delta_{\mathbf{E T}}$ becomes infinite, leading to a physically unrealistic unbounded melt region while $\delta_{\mathbf{2} \mathbf{L}}(\pi / 2)=0.1325$, leading to a finite melt region.

Using the improved boundary condition $\mathbf{u}(\mathbf{y})=-\sin (\theta)$ to determine the velocity $\mathbf{u}(\mathbf{y})$ in conjunction with the two-layer approach leads to a further improvement in the estimate of the melt-layer thickness. If $\delta_{\mathbf{2 M}}(\theta)$ denotes the melt-layer thickness using this modified two-layer approach, then the relations $\delta_{\mathbf{2 M}}<\delta_{\mathbf{2 L}}<\delta_{\mathbf{M}}<\delta_{\mathbf{E T}}<\delta_{\mathbf{E T 0}}$ hold among the different melt-layer expressions. Using the same values for $\sigma$, Ste, $\mathrm{Pe}$, and $\beta$, yields $\delta_{\mathbf{2 M}}(\pi / 2)=0.1306$. If the radius of the sphere is reduced, $\sigma$, Ste, and $\mathrm{Pe}$ increase while $\beta$ decreases. The relative percentage errors, $100 \times\left|\delta_{\mathbf{2} \mathbf{M}}(\theta)-\delta_{\mathbf{2} \mathbf{L}}(\theta)\right| /\left|\delta_{\mathbf{2} \mathbf{L}}(\theta)\right|$ range between 0.07 and 1.46 for $0 \leq \theta \leq \pi / 2$.

\section{References}

1. Emerman, S. H.,Turcotte, D. L.: Stokes's problem with melting. Int. J. Heat Mass Transfer. Vol. 26, No. 11 (1983) 1625-1630.

2. Mukherjee, A., Stevens, J., G.: Heat transport in Stokes' problem with melting: A two-layer approach. Int. J. of Heat Mass Transfer. to appear.

3. Rasmussen, N. C., Yellin, J., Kleitman D. J., and Stewart R. B.: Nuclear power: can we live with it? Tech. Rev. 81 (1979) 32-46. 